\title{
Reaction of 1,1,3,3-Tetraoxa-2,2-dibromo-4,4,5,5,6,6-hexafluoro-
}

\section{1,3-dithiacyclohexane with Pyrazine and Hexamethyl Phosphoramide:}

\section{Unexpercted Co-crystals through halogen bonding}

Shizheng Zhu ${ }^{*}$, Huiling Jiang, Jingwei Zhao and Zhanting $\mathrm{Li}^{*}$

The State Key Laboratory of Organofluorine Chemistry, Shanghai Institute of Organic Chemistry, Chinese Academy of Sciences

\section{Supporting Information}

Table 1. Selected Bond Data of Compounds 1, 5 and 6.

\begin{tabular}{|c|c|c|}
\hline Compound & Bond Length $(\AA)$ & $\begin{array}{c}\text { Halogen or Hydrogen } \\
\text { Bond Distance }(\AA) \text { and } \\
\text { Angles }\left(\left(^{\circ}\right)\right.\end{array}$ \\
\hline $\begin{array}{c}\mathrm{F}_{2}{ }^{2} \mathrm{C}-{ }^{1} \mathrm{SO}_{2} \\
\mathrm{~F}_{2}{ }^{3} \mathrm{C}_{1}^{1} \mathrm{C}_{-}^{-\mathrm{H}} \\
\mathrm{F}_{2}{ }^{4} \mathrm{C}-{ }^{2} \mathrm{SO}_{2}\end{array}$ & $\begin{array}{ll}C^{1}-B r & 1.915 \\
C^{1}-S^{1} & 1.800 \\
C^{1}-S^{2} & 1.800 \\
C^{2}-S^{1} & 1.859 \\
C^{4}-S^{2} & 1.859\end{array}$ & $\begin{array}{cc}\mathrm{C}-\mathrm{Br} \cdots \mathrm{O} & 3.364 \\
& 154.3 \\
& \\
\mathrm{C}-\mathrm{H} \cdots \mathrm{O} & 2.543 \\
& 138.9\end{array}$ \\
\hline 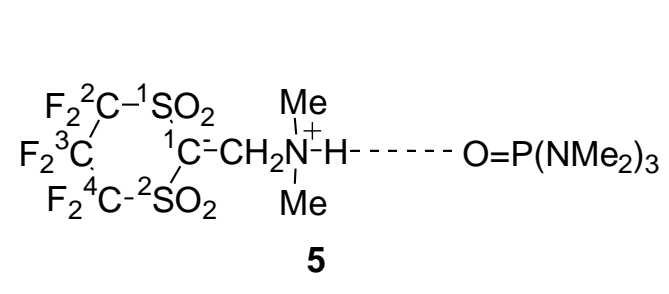 & $\begin{array}{ll}C^{1}-S^{1} & 1.676 \\
C^{1}-S^{2} & 1.680 \\
C^{2}-S^{1} & 1.843 \\
C^{4}-S^{2} & 1.851\end{array}$ & $\begin{array}{cc}\mathrm{N}-\mathrm{H} \cdots \mathrm{O} & 2.603 \\
\mathrm{~d}_{\mathrm{H}} \cdots \mathrm{O} & 1.689 \\
& 168.7\end{array}$ \\
\hline${ }_{6}^{\mathrm{F}_{2}^{2} \mathrm{C}-{ }^{1} \mathrm{SO}_{2} \mathrm{Br}^{1} \ldots-{ }^{3} \mathrm{Br}^{+} \ldots . .}$ & $\begin{array}{ll}\mathrm{C}^{1}-\mathrm{Br}^{1} & 1.945 \\
\mathrm{C}^{1}-\mathrm{Br}^{2} & 1.954 \\
\mathrm{C}^{1}-\mathrm{S}^{1} & 1.805 \\
\mathrm{C}^{1}-\mathrm{S}^{2} & 1.804 \\
\mathrm{C}^{2}-\mathrm{S}^{2} & 1.854 \\
\mathrm{C}^{4}-\mathrm{S}^{2} & 1.849\end{array}$ & $\begin{array}{rc}{ }^{1} \mathrm{C}-\mathrm{Br}^{1} \cdots{ }^{3} \mathrm{Br}^{+} & 3.011 \\
& 175.3 \\
& \\
{ }^{1} \mathrm{C}-\mathrm{Br}^{2} \cdots{ }^{3 \mathrm{~A}} \mathrm{Br}^{-} & 3.070 \\
& 173.7\end{array}$ \\
\hline
\end{tabular}




\section{Crystallographic data for compound 5:}
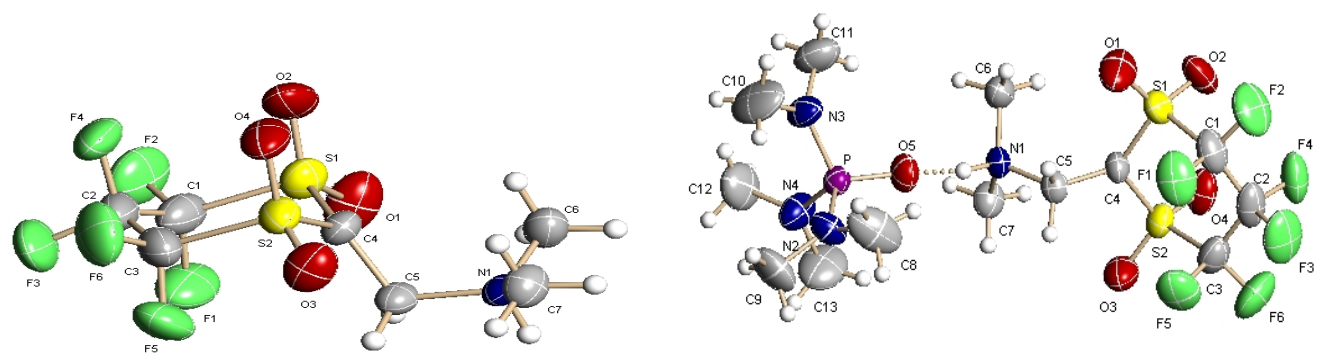

Formula $\mathrm{C}_{13} \mathrm{H}_{27} \mathrm{~N}_{4} \mathrm{O}_{5} \mathrm{~F}_{6} \mathrm{PS}_{2} ; \mathrm{Mr}=528.48 ;$ moloclinic, space group $\mathrm{P} 2(1) / \mathrm{c}$; temperature 293(2)(K); $a=13.6434(11) \AA, b=14.8362(12) \AA, c=13.1386(11) \AA, a=$ $90^{\circ}, \beta=118.38(10)^{\circ}, Y=90^{\circ} ; \quad V=2339.9(3) \AA^{3} ; Z=4, D c=1.500 \mathrm{mg} / \mathrm{m}^{3} ;$ absorption coefficient $0.374 \mathrm{~mm}^{-1} ; \mathrm{F}(000)=1096 ; 1.70<\theta<28.29$; reflections collected / unique $13998 / 5446$ [R(int)=0.0570]; absorption correction sadabs; transmission 1.000 mix$0.73661_{\min }$, final $\mathrm{R}$ indices $\mathrm{R} 1=0.0475, \mathrm{wR} 2=0.1135 .{ }^{1} \mathrm{H}-\mathrm{NMR}\left(\mathrm{CDCl}_{3}\right): \delta 2.70$ (broad, $\left.{ }^{+} \mathrm{NH}\right), 2.68(18 \mathrm{H}, \mathrm{s}, \mathrm{HMPA}), 2.63\left(6 \mathrm{H}, \mathrm{s},{ }^{+} \mathrm{NMe}_{2}\right), 1.91\left(2 \mathrm{H}, \mathrm{m}, \mathrm{CH}_{2}\right)$. ${ }^{19} \mathrm{~F}-\mathrm{NMR}\left(\mathrm{CDCl}_{3}\right): \delta-113.9\left(1 \mathrm{~F}^{\mathrm{a}} \mathrm{C},{ }^{2} J_{\mathrm{F} \mathrm{F}}^{\mathrm{a}} \mathrm{e}=276 \mathrm{~Hz}\right),-117.8\left(2 \mathrm{~F}^{\mathrm{a}} \mathrm{S},{ }^{2} J_{\mathrm{F} \mathrm{F}}^{\mathrm{a}}=228 \mathrm{~Hz}\right)$, $-123.2\left(2 \mathrm{~F}^{\mathrm{e}} \mathrm{S},{ }^{2} J_{\mathrm{F} \mathrm{F}}^{\mathrm{a}} \mathrm{e}=228 \mathrm{~Hz}\right)-135.0\left(1 \mathrm{~F}^{\mathrm{e}} \mathrm{C},{ }^{2} J_{\mathrm{F} \mathrm{F}}^{\mathrm{a}}=276 \mathrm{~Hz}\right) . \mathrm{MS}(\mathrm{m} / \mathrm{z}, \%)$ : 414 $\left(\mathrm{M}^{+}-\mathrm{CF}_{2} \mathrm{SO}_{2}, 8.15\right), 344\left(\mathrm{M}^{+}-\mathrm{CF}_{2} \mathrm{SO}_{2}-\mathrm{CH}_{2} \mathrm{O}, 6.67\right), 311\left(\mathrm{HMPA}+\mathrm{SCF}_{2} \mathrm{CF}_{2}{ }^{+}, 5.92\right)$, $215\left(\mathrm{H}\left(\mathrm{CF}_{2}\right)_{3} \mathrm{SO}_{2}{ }^{+}, 1.60\right), 179\left(\mathrm{HMPA}^{+}, 53.28\right), 135\left({ }^{+} \mathrm{P}(\mathrm{O})\left(\mathrm{NMe}_{2}\right)_{2}, 100\right), 100\left(\mathrm{C}_{2} \mathrm{~F}_{4}{ }^{+}\right.$, 22.04), $92\left(\mathrm{HP}(\mathrm{O})\left(\mathrm{NMe}_{2}\right)_{2}, 24.76\right), 44\left(\mathrm{Me}_{2} \mathrm{~N}^{+}, 84.69\right)$. FT-IR $(\mathrm{KBr}): \mathrm{v}=2925(\mathrm{~m})$, 2456 (m), 1348 (s), 1142 (s), 990 (s), 747 (s) $\mathrm{cm}^{-1}$.

\section{Crystallographic data for compound 6:}

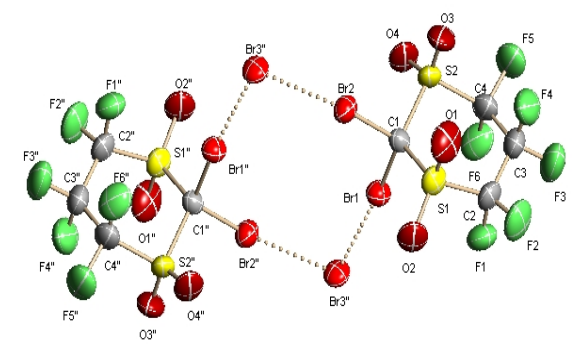

Formula $\mathrm{C}_{10} \mathrm{H}_{18} \mathrm{Br}_{3} \mathrm{~F}_{6} \mathrm{~N}_{3} \mathrm{O}_{5} \mathrm{PS}_{2} ; \mathrm{Mr}=709.09$; triclinic, $\mathrm{P}-1$; temperature 293(2)(K); a $=7.7985(7) \AA, b=12.0911(10) \AA, c=13.2824(11) \AA, \alpha=106.716(2)^{\circ}, \beta=99.849(2)^{\circ}$, $\mathrm{V}=90.897(2)^{\mathrm{o}} ; \mathrm{V}=1179.03(17) \quad \AA^{3} ; \mathrm{Z}=2, \mathrm{Dc}=1.997 \mathrm{mg} / \mathrm{m}^{3} ;$ absorption coefficient $5.450 \mathrm{~mm}^{-1} ; \mathrm{F}(000)=690 ; 1.63<\theta<27.00 ;$ reflections collected / unique $6962 / 4976$ $[\mathrm{R}(\mathrm{int})=0.1648]$; absorption correction empirical; transmission $1.000 \mathrm{mix}^{-} 0.065_{\mathrm{min}}$. 
final $\mathrm{R}$ indices $\mathrm{R} 1=0.0694, \mathrm{wR} 2=0.1678 .{ }^{1} \mathrm{H}-\mathrm{NMR}\left(\mathrm{CDCl}_{3}\right)$ : $\delta 2.64$ (s, HMPA ). ${ }^{19} \mathrm{~F}-\mathrm{NMR}\left(\mathrm{CDCl}_{3}\right): \quad \delta-114.0\left(1 \mathrm{~F}^{\mathrm{a}} \mathrm{C},{ }^{2} J_{\mathrm{F} \mathrm{F}}^{\mathrm{a}}{ }^{\mathrm{e}}=282 \mathrm{~Hz}\right),-118.9\left(2 \mathrm{~F}^{\mathrm{a}} \mathrm{S},{ }^{2} J_{\mathrm{F} \mathrm{F}}^{\mathrm{a}}=248 \mathrm{~Hz}\right)$, $-124.3\left(2 \mathrm{~F}^{\mathrm{e}} \mathrm{S},{ }^{2} J_{\mathrm{F} \mathrm{F}}^{\mathrm{a}}=248 \mathrm{~Hz}\right),-135.8\left(1 \mathrm{~F}^{\mathrm{e}} \mathrm{C},{ }^{2} J_{\mathrm{F} \mathrm{F}}^{\mathrm{a}}=282 \mathrm{~Hz}\right)$. FT-IR $(\mathrm{KBr}): v=2925$ (m), 2854 (m), 1465 (s), 1348 (s), 1304 (s), $1141(\mathrm{~s}), 1045$ (s), 747 (s), 600 (s), 536 (s) $\mathrm{cm}^{-1}$. 